\title{
Role of L-asparaginase in acute lymphoblastic leukemia: focus on adult patients
}

REVIEW

This article was published in the following Dove Press journal:

Blood and Lymphatic Cancer:Targets and Therapy

28 June 2012

Number of times this article has been viewed

\author{
Michael E Rytting \\ Department of Pediatrics and \\ Leukemia, University of Texas MD \\ Anderson Cancer Center, Houston, \\ TX, USA
}

\begin{abstract}
Asparaginase preparations deplete asparagine in acute lymphoblastic leukemia (ALL) blasts. Asparaginase in its various forms is an important component of treatment regimens for pediatric ALL. Recently, interest and use of asparaginase in adult patients with ALL has increased, particularly in young adults. There is much less information on asparaginase use and toxicity in adult compared with pediatric populations. This review surveys prior published studies of the three most commonly used asparagine preparations as used in adult patients, and discusses important toxicities encountered in adult patients who receive asparaginase preparations.
\end{abstract}

Keywords: asparaginase, leukemia, adults, children

\section{Introduction}

In 1953, Kidd noted the remarkable observation that guinea pig serum had antileukemia activity in mice. ${ }^{1}$ The cause of this antileukemia activity was elucidated by Broome in 1961 and attributed to depletion of asparagines. ${ }^{2}$ Quite rapidly, investigators confirmed the ability of Escherichia coli L-asparaginase to bring about remissions in children with acute lymphoblastic leukemia (ALL). ${ }^{3-6}$ L-asparaginase was initially envisioned as a targeted antileukemia drug, able to kill malignant lymphoblasts while not harming the normal cells of the patient, but this optimism proved to be short-lived; papers on anaphylactic shock and pancreatitis in conjunction with L-asparaginase rapidly appeared. ${ }^{7,8}$ Other toxicities of the drug have subsequently become well known.

L-asparaginase was incorporated into treatment protocols for children with ALL in the 1970s, and the drug has remained a consistent component of pediatric ALL treatment regimens. There are several excellent reviews and a multitude of published trials that detail the use of L-asparaginase and its derivatives, Erwinia asparaginase (Erwinia) and pegylated asparaginase, in pediatric patients. ${ }^{10-13}$ The use of L-asparaginase in adult patients with ALL is also prominent, although L-asparaginase is still not universally employed in standard adult ALL regimens, most notably the hyper CVAD (cyclophosphamide, vincristine, adriamycin, dexamethasone) regimen. ${ }^{14,15}$ Still, there is a surge of interest in the use of asparaginases, particularly PEG-asparaginase, in adults with ALL. Erwinia asparaginase is also generating interest, being principally substituted in patients with anaphylactic reactions to L-asparaginase or PEG-asparaginase.

This review describes the present use of asparaginase preparations in adults with ALL and the toxicities currently reported in this older group of patients. Similar to
Correspondence: Michael E Rytting Department of Pediatrics and Leukemia, University of Texas MD Anderson Cancer Center, I5I5 Holcombe Blvd, Unit 87, Houston, TX, USA

Email mrytting@mdanderson.org 
pediatric regimens, adult treatment protocols for ALL use initial induction treatment to induce remission quickly. Single-institution studies as well as multicenter studies from Europe and the United States all incorporate asparaginase into ALL regimens. However, not all adult ALL regimens include L-asparaginase, and intensive regimens not containing L-asparaginase can very successfully induce a complete response in adult patients. ${ }^{16}$ Along that line, Kantarjian et al reported successful induction using VAD (vincristine, adriamycin, dexamethasone) or cytoxan VAD in standard-risk adult ALL patients without using any asparaginase. ${ }^{15}$ Subsequent development of the hyper CVAD regimen at the MD Anderson Cancer Center did not incorporate L-asparaginase but did produce high complete response rates. ${ }^{14,15}$

\section{L-asparaginase}

Asparaginase is currently used in three forms, ie, asparaginase manufactured from $E$. coli, asparaginase derived from Erwinia chrysanthemi, and L-asparaginase conjugated with polyethylene glycol (PEG). The chemistry and mechanism of action of asparaginase preparations has been detailed in multiple reviews. ${ }^{10,12,17,18}$ Briefly, cells require asparagine to proceed through the $\mathrm{G} 1$ cycle of cell division. Asparagine is important in protein, DNA, and RNA synthesis. Normal human tissues can synthesize asparagine via asparagine synthetase, but many malignant cells cannot do so. Thus, asparagine is an essential amino acid for tumor cells, but not for normal cells, and this can be exploited in designing treatment regimens for certain cancers. On the other hand, some malignant tumor cells demonstrate increased expression of asparagine synthetase, perhaps through endoplasmic reticulum stress pathways or amino acid response pathways. As expected, increased expression of asparagine synthetase corresponds to decreased sensitivity to asparaginase.

\section{L-asparaginase from $E$. coli}

L-asparaginase derived from $E$. coli has been used in acute leukemia therapy since the 1960s and is the most studied form of asparaginase. It has a fascinating history. The drug may be administered as an intravenous infusion or as an intramuscular injection. The drug has also been given to patients intrathecally. ${ }^{19}$ Initial studies of L-asparaginase in ALL evaluated the drug when it was given daily for as many as 28 consecutive days. ${ }^{20}$ The half-life of the L-asparaginase enzyme and the enzymatic activity of asparaginase are similar, and do not appear to vary with repeated doses nor with the dose administered. The half-life of enzymatic activity is approximately 1.24 days in children. ${ }^{21}$ In adult patients with leukemia, the drug is administered intravenously or intramuscularly, usually at doses of $25,000 \mathrm{U} / \mathrm{m}^{2}$ given once or in regimens where $6000 \mathrm{U} / \mathrm{m}^{2}$ are given every other day for six to nine doses. Depletion of serum asparagine is complete in the majority of children treated on a schedule of 2500-5000 IU/ $/ \mathrm{m}^{2}$ given every third day for eight doses. L-asparaginase does not cross the blood-brain barrier, but the drug does successfully deplete asparagine in the spinal fluid. $^{22,23}$

\section{Erwinia asparaginase}

The Erwinia asparaginase in use currently is produced by E. chrysanthemi. Erwinia, like asparaginase, may be administered intramuscularly or intravenously. Erwinia is currently approved for the treatment of leukemia in the United Kingdom. In the United States, an ongoing trial in children and adult patients with prior allergic reactions to other asparaginase preparations is finalizing information on the tolerability of Erwinia in order to obtain approval of the drug. The dose of Erwinia in this trial is 10,000 IU intramuscularly or intravenous every other day for six doses. The drug has previously been administered at similar doses and schedules as L-asparaginase, and several head-to-head comparisons with L-asparaginase have shown decreased efficacy for Erwinia as summarized by Pieters et al. ${ }^{24}$ Erwinia has a decreased half-life compared with L-asparaginase, and the drug must be given at higher doses and more frequently than L-asparaginase in order to deplete asparagine completely. ${ }^{21}$ Asparagine levels also recover more quickly in patients receiving Erwinia, at least in children. ${ }^{25}$ Erwinia has been demonstrated to be effective in patients with severe allergic reactions to L-asparaginase, and that is the most common clinical setting in which clinicians use Erwinia. ${ }^{26}$ Another application of Erwinia might be in patients with silent antibody formation to $E$. coli asparaginase preparations. These patients rapidly clear asparaginase unbeknownst to their physicians. Erwinia asparaginase may circumvent this rapid clearance and allow for better asparagine depletion. ${ }^{26}$ Currently, testing for silent antibodies is not routinely done on most protocols.

\section{PEG-asparaginase}

Pegylation of $E$. coli-derived asparaginase increases the circulation time for the asparaginase enzyme and decreases the immunogenicity of asparaginase. PEG-asparaginase pharmacokinetics are otherwise similar to those of native asparaginase. ${ }^{12}$ The drug shows one-compartment distribution, a monophasic half-life, and a single elimination phase. The prolonged halflife is the most important characteristic of the drug. 
PEG-asparaginase has a half-life of about 6 days, considerably longer than the E. coli or Erwinia preparations. A single dose of PEG-asparaginase may be substituted for a series of injections of nonpegylated asparaginase. Antibodies formed against asparaginase or PEG alter the clearance of the drug. Patients who have had overt allergic reactions to L-asparaginase have a shorter half-life for PEG-asparaginase than patients without prior reactions to asparaginase preparations. ${ }^{10}$ In addition to overt allergy, a shortened half-life may be encountered in the setting of occult antibody formation. ${ }^{26}$ Patients with silent antibodies might benefit from a switch to Erwinia asparaginase or a more frequent dosing of PEG-asparaginase. ${ }^{26}$ Patients with antibodies to PEG also clear the drug more rapidly than patients without antibodies to PEG, as demonstrated by Armstrong et al. ${ }^{27}$ Interestingly, development of PEG antibodies could be on the increase in the general population, because PEG is used in a variety of commonly encountered products, including cosmetics and processed foods. Routine testing for antibodies to PEG is not done, but might be worthwhile in newly diagnosed adult patients who are to receive regimens that contain PEG-asparaginase.

\section{E. coli asparaginase in adult trials}

Adult ALL protocols routinely incorporate asparaginase as part of therapy. As in pediatric trials, the intensity and schedule of asparaginase treatment vary markedly. As opposed to pediatrics, not all successful adult ALL therapies use asparaginase in treating ALL. Notably, the hyper CVAD regimen used at MD Anderson Cancer Center initially did not employ asparaginase and has excellent induction results and eventfree survival in young adults with ALL. Still, most trials performed on adult ALL patients from the early 1970s demonstrated that a moderate fraction of patients could be cured using complex regimens that employed asparaginase. Results from several large trials are shown in Table 1. L-asparaginase was typically given every other day for several doses, biweekly, or the drug was given on consecutive days for up to 2 weeks. ${ }^{31}$ The dose in most protocols ranged from 5000 to $10,000 \mathrm{IU} / \mathrm{m}^{2}$, given on multiple days.

Table I Doses of Escherichia coli asparaginase in adult trials

\begin{tabular}{|c|c|c|c|}
\hline Reference & $\mathbf{n}$ & Asparaginase dose & Overall survival \\
\hline Schauer et $\mathrm{al}^{28}$ & 72 & $\begin{array}{l}\text { L-asparaginase } \\
200 \mathrm{IU} / \mathrm{kg}\end{array}$ & $50 \%$ \\
\hline Lister et $\mathrm{al}^{29}$ & 51 & L-asparaginase & $20 \%$ \\
\hline Marcus et $\mathrm{al}^{30}$ & 69 & $\begin{array}{l}\text { L-asparaginase } \\
10,000 \mathrm{IU} / \mathrm{m}^{2}\end{array}$ & $32 \%$ \\
\hline Hoelzer et $\mathrm{al}^{34}$ & 162 & $\begin{array}{l}\text { L-asparaginase } \\
5000 \mathrm{lU} / \mathrm{m}^{2}\end{array}$ & $44 \%$ \\
\hline
\end{tabular}

In the 1980s, results from large adult ALL trials that employed asparaginase were reported and showed minimal improvement in event-free survival. Doses continued to vary from one protocol to another. A randomization between 4 or 12 doses of L-asparaginase $10,000 \mathrm{IU} / \mathrm{m}^{2}$ during induction failed to show any improvement or worsening in disease-free survival in a large Medical Research Council trial. ${ }^{32}$ L-asparaginase was generally used early in therapy, in induction or consolidation, or both. Protocols were disparate on the timing of asparaginase, with several studies starting L-asparaginase on the first day to the fifth day of induction, while other protocols did not introduce L-asparaginase until approximately day 100 of therapy or later. The dose of L-asparaginase varied widely between protocols in the 1980s. Commonly used doses were in the range of 6000$12,000 \mathrm{IU} / \mathrm{m}^{2}$ given on consecutive or alternate days. ${ }^{16,33,34}$ A few protocols dosed L-asparaginase based on weight, with a dose range of 200-500 IU/kg. ${ }^{35}$ Such weight-based dosing might prove toxic given the current epidemic of obesity. Interestingly, one protocol was able to administer 60,000 IU of L-asparaginase intravenously three times weekly for 2 weeks. ${ }^{36}$ Still, when compared with pediatric protocols, adult studies in the 1980s tended to introduce asparaginase later in induction, and exposure to L-asparaginase was considerably briefer than what is typically seen in pediatric trials. In GMALL 04/89, Ludwig et al used L-asparaginase $10,000 \mathrm{IU} / \mathrm{m}^{2}$ following intravenous methotrexate during weeks 13, 15, and 17 for a total of three doses, with a significant improvement in clinical complete response compared with their prior regimen. ${ }^{37}$

In the 1990s, studies in adult patients continued apace. L-asparaginase dosing was much more uniform in reported studies, with 6000-10,000 IU/m² being generally used. However, the frequency and timing of L-asparaginase administration remained far from uniform. Ribera et al reported perhaps the most intensive use of L-asparaginase, with 16 doses of $10,000 \mathrm{IU} / \mathrm{m}^{2}$ in induction and consolidation followed by $20,000 \mathrm{IU} / \mathrm{m}^{2}$ given twice in delayed intensification. ${ }^{38}$ The very large international trial in adults with ALL conducted by the Medical Research Council/Eastern Cooperative Oncology Group used L-asparaginase at the relatively conventional dose of $10,000 \mathrm{IU} / \mathrm{m}^{2}$ initially, then changed to $10,000 \mathrm{IU}$ as a flat dose on days $17-28$ of induction. ${ }^{39}$ Three more doses of L-asparaginase 10,000 IU were given in the first 3-4 weeks following induction with no further asparaginase delivered in this international trial. The timing of L-asparaginase therapy in the different published regimens remained variable. While use in induction and consolidation was the norm, the timing 
of asparaginase therapy in induction ranged from early in the first week of therapy to as late as the ninth week of treatment. Again in the 1990s, the hyper CVAD regimen with no use of asparaginase achieved an excellent remission rate and a longterm overall survival rate comparable with those regimens for adults that contained L-asparaginase. Similarly, a large Japanese study randomized adults to therapy with or without L-asparaginase and found no significant difference in outcome between the two groups. ${ }^{40}$

Since 2000, L-asparaginase has been slowly replaced by the pegylated form of asparaginase, mostly due to the notable convenience of dosing. Still, there have been significant trials that incorporated L-asparaginase and reported in the last decade, particularly in younger adults. The Dutch-Belgian Hemato-Oncology Cooperative Group evaluated a pediatricbased regimen for patients aged $17-40$ years. ${ }^{41}$ This protocol used 21 scheduled doses of L-asparaginase at the conventional dose of $6000 \mathrm{IU} / \mathrm{m}^{2}$. Nine doses of L-asparaginase were administered starting immediately after a prednisone prephase, and further doses occurred later in therapy, similar to the design of pediatric therapy. In a much larger trial, Huguet et al administered an intensive ALL treatment regimen to patients aged 15-60 years. L-asparaginase was introduced on day 8 of induction, 2 weeks after a prednisone window. ${ }^{42}$ The dose of L-asparaginase was $6000 \mathrm{IU} / \mathrm{m}^{2}$ for six doses in induction, with doses of $10,000 \mathrm{IU} / \mathrm{m}^{2}$ given approximately every 3 weeks in consolidation for six doses. A repeat course of $6000 \mathrm{IU} / \mathrm{m}^{2}$ given every other day for six doses was given in delayed intensification. In the above study, patients over 45 years of age had an unacceptable toxic death rate. The Spanish group used L-asparaginase successfully in a trial for young adults up to 30 years of age with ALL. ${ }^{43}$ L-asparaginase was started on day 10 of therapy. Fifteen doses of L-asparaginase $10,000 \mathrm{IU} / \mathrm{m}^{2}$ were given during induction and consolidation, and one dose of 20,000 IU/ $/ \mathrm{m}^{2}$ was given in the reinduction phase. Outcomes were similar between adolescent patients and adults on this regimen up to the age of 30 years. Much less L-asparaginase was used by Labar et al in their randomized trial. ${ }^{44} \mathrm{~L}$-asparaginase was not given in induction, but rather approximately three doses of $10,000 \mathrm{IU} / \mathrm{m}^{2}$ were given starting at day 66 of therapy.

\section{Erwinia asparaginase in adult trials}

Erwinia asparaginase has been used in the past in at least two forms, ie, E. chrysanthemi and E. carotavora. Currently, E. chrysanthemi is used. An extensive search of the literature showed no evaluations of Erwinia asparaginase as frontline therapy for adult ALL patients. However, the drug has been studied extensively as second-line or later therapy, and there are multiple ongoing studies that employ Erwinia asparaginase in second-line or third-line treatment of ALL in pediatrics. The use of Erwinia asparaginase in adults with ALL parallels pediatric use, with the drug being substituted as a second-line or third-line form of asparaginase. In pediatric trials, the drug is routinely given at $10,000-20,000 \mathrm{IU} / \mathrm{m}^{2}$ two or three times per week. The drug is a regular substitution for adult and pediatric patients who are allergic to L-asparaginase or PEG-asparaginase preparations. In adult trials, Erwinia asparaginase is most often given every other day, reflecting the short half-life of the drug, with the most frequent dose being $10,000 \mathrm{IU} / \mathrm{m}^{2}{ }^{21,24}$ A current clinical trial of Erwinia asparaginase is enrolling both children and adults who have had allergic reactions to PEG-asparaginase. This trial may lead to approval of Erwinia asparaginase in the United States. At the present time, there is no significant evidence that demonstrates superior clinical efficacy for intramuscular versus intravenous Erwinia, nor is there any conclusive evidence that one route is more likely to cause toxicity than another.

\section{PEG-asparaginase in adult trials}

Due to its prolonged half-life and prolonged depletion of asparagine, PEG-asparaginase has proven to be attractive as an alternative to the other asparaginase forms. In addition, pegylation of asparaginase may lead to fewer allergic reactions. PEG-asparaginase has been studied in adult patients since at least the early 1980s. In 1981, Park et al evaluated the pharmacology of a PEG adduct of asparaginase in four adult patients. ${ }^{45}$ This initial pharmacology study was followed by a larger Phase I/II trial that studied the pharmacology of PEG-asparaginase in 37 adult patients at the MD Anderson Cancer Center. ${ }^{46}$ Doses of $250-8000 \mathrm{IU} / \mathrm{m}^{2}$ were evaluated. An open-label Phase II trial of PEGasparaginase was performed in $1995 .{ }^{47}$ This trial used $2000 \mathrm{IU} / \mathrm{m}^{2}$ given every 2 weeks as a single agent in 18 adults with ALL. PEG-asparaginase 2000-2500 IU/m² given intravenously or intramuscularly is the standard adult dose. Some current studies impose a cap at 3750 IU per dose. Given the prolonged half-life of the drug, a 2-3week interval between doses may be effective. A handful of studies have evaluated PEG-asparaginase in combination chemotherapy regimens for adults with newly diagnosed or relapsed ALL. The CALGB (Cancer and Leukemia Group B) study 9511 incorporated PEG-asparaginase $2500 \mathrm{IU} / \mathrm{m}^{2}$, with a cap at $3750 \mathrm{IU}$, for four doses. ${ }^{48}$ 
Aguayo et al used PEG-asparaginase at $2500 \mathrm{IU} / \mathrm{m}^{2}$ (maximum dose $3750 \mathrm{IU}$ ) in a combination regimen for relapsed ALL. ${ }^{49}$ The Berlin-Frankfurt-Munster group used PEG-asparaginase in protocol 05/93 and allowed intrapatient dose escalation according to asparaginase levels. A $500-1000 \mathrm{IU} / \mathrm{m}^{2}$ dose escalation was assessed, and the escalation was well tolerated. ${ }^{50}$

Many adult protocols are attempting to recapitulate pediatric-based therapy for de novo ALL in an attempt to improve treatment results in younger adult patients. As pediatric trials have moved to PEG-asparaginase, so too has PEG-asparaginase use in the young adult population become prominent. Douer et al has successfully used a dose of $2000 \mathrm{IU} / \mathrm{m}^{2}$ of PEG-asparaginase on day 16 of induction for adults up to age 55 years with newly diagnosed ALL. ${ }^{51}$ At the University of Texas MD Anderson Cancer Center, adults up to the age of 40 years are given $2500 \mathrm{IU} / \mathrm{m}^{2}$, with a total of seven doses administered during the first 6 months of treatment; the PEG-asparaginase dose is not capped..$^{52}$ The CALGB has started a multi-institution evaluation of pediatric-based therapy in young adults with ALL that also uses PEG-asparaginase $2500 \mathrm{IU} / \mathrm{m}^{2}$ administered throughout treatment, as is done in pediatric-based ALL treatment regimens.

\section{Asparaginase toxicities in adult trials Pancreatitis}

All three asparaginase preparations have been implicated in causing pancreatitis. In children, a randomized trial of PEG-asparaginase versus L-asparaginase for standard risk ALL showed no significant difference in the incidence of grade 3-4 pancreatitis. ${ }^{53}$ In children, the incidence of pancreatitis with asparaginase may increase with patient age, which implies that adults should have higher rates of pancreatitis than children. ${ }^{54}$ This has not been confirmed in several studies of adults treated with pediatric-based regimens. In the studies by Huguet et al and Ribera et al, no clinically significant cases of pancreatitis were reported. ${ }^{42,43}$ Similarly, no pancreatitis was seen in 26 adult patients studied with escalating doses $\left(500-1000 \mathrm{IU} / \mathrm{m}^{2}\right)$ of PEG-asparaginase ${ }^{50}$ nor is pancreatitis noted to be a toxicity in several other adult trials. ${ }^{41,47,55}$ However, in general, the incidence of pancreatitis in conjunction with asparaginase is generally assumed to be about $5 \%-10 \% .^{13,53}$ Generally, if a combination of clinical plus radiographic or biochemical findings confirm pancreatitis is present, further use of asparaginase is not recommended.

\section{Thrombosis and hemorrhage}

Thrombus formation is a significant worry when using asparaginase in adult patients. The incidence of thrombotic complications in patients on asparaginase-containing regimens varies from less than $10 \%$ to greater than $30 \% .{ }^{56}$ The incidence of thrombus formation increases with the age of the patient population. In adults, central lines are frequently placed in the arm rather than into a subclavian vein. The smaller caliber vein may contribute to thrombus formation. Pooled data on PEG-asparaginase, published in abstract form, indicates thrombus formation occurs in approximately $5 \%-15 \%$ of adult patients when PEG-asparaginase is used..$^{52}$ Further information is needed concerning PEG-asparaginase and thrombosis, especially in older adults; more extensive information in children so far has shown that PEGasparaginase is not associated with increased thrombosis. ${ }^{57-60}$ The timing of thrombus formation is generally early in therapy; the GOELAMS study found a 9.3\% incidence during induction, ${ }^{61}$ possibly related to confounding factors, such as hypercoagulability due to the active leukemia and placement of new central lines. The type of induction corticosteroid also influences thrombus formation during asparaginase therapy, with prednisone being associated with a higher risk of thrombosis. ${ }^{62,63}$ The incidence of thrombotic events appears to be about equivalent when comparing Erwinia asparaginase and L-asparaginase. After the diagnosis of an asparaginaseinduced thrombus, low molecular weight heparin has been used with success, and asparaginase therapy may be restarted in patients who have had adequately treated thrombotic events. ${ }^{56}$ The value of antithrombin III infusions is not clear, though the GOELAMS investigators did show a decrease in thrombosis when prophylactic antithrombin III infusion was used. ${ }^{61}$

Serious hemorrhagic complications secondary to depleted clotting factors are less frequent than thrombotic events during asparaginase therapy ${ }^{61}$ Fibrinogen levels frequently fall during therapy, but the issue of fibrinogen replacement at an agreed upon fibrinogen level has not been settled; a fibrinogen level less than $1 \mathrm{~g} / \mathrm{L}$ frequently triggers replacement therapy.

\section{Liver toxicity}

As noted in prior reviews, there are limited specific data on liver toxicity and asparaginase use. Most ALL regimens employ several potentially hepatotoxic drugs, such as high dose corticosteroids, vinca alkaloids, anthracyclines and antimetabolites. In addition, adult leukemia patients often receive antifungal prophylaxis with azoles, and adults have 
other causes of hepatic toxicity, including alcohol. Quantifying the hepatic toxicity of asparaginase in such patients is difficult. Early evaluations of L-asparaginase at doses as high as $1000 \mathrm{IU} / \mathrm{kg}$ caused hepatic dysfunction in up to $75 \%$ of patients. ${ }^{64}$ Lower doses of asparaginase, including PEG-asparaginase, result in less hepatic toxicity, but liver toxicity is still prominent. ${ }^{47,51,52,56}$ In the great majority of adult patients, these abnormalities resolve over time.

\section{Immunological reactions to asparaginase}

Hypersensitivity reactions to asparaginase preparations are not rare and can be serious. The risk of a severe allergic reaction changes depending on prior exposures and concomitant medications (corticosteroids). In induction regimens that use high-dose steroids, allergic reactions to asparaginase are infrequent. In 1500 adults treated on the Medical Research Council UKALL XII/Eastern Cooperative Oncology Group E2993, a 90\% induction success was reported for a welltolerated induction regimen that employed high-dose prednisone and multiple injections of L-asparaginase. ${ }^{39}$ There was no mention of allergic reactions to asparaginase. Similarly, multiple authors have noted no severe allergic reactions to PEG-asparaginase in adults during asparaginase therapy. ${ }^{41,50,52,65,66}$ It seems likely that with repeated exposures and while patients are not receiving corticosteroids, allergic reactions become more prominent and probably occur in approximately $5 \%-10 \%$ of patients. ${ }^{67,68}$ An allergic reaction rate of $10 \%$ or greater is more in keeping with the larger pediatric experience with asparaginase. A purported advantage of PEG-asparaginase is that it is less immunogenic than L-asparaginase. Still, severe allergic reactions clearly occur in patients who get repeated doses of PEG-asparaginase. ${ }^{51,52}$ Patients who have allergic reactions to L-asparaginase or PEG-asparaginase may be successfully treated with Erwinia asparaginase. In the main, antibodies to PEG-asparaginase and L-asparaginase do not cross-react with Erwinia. Allergic reactions to Erwinia can occur in up to one third of children. ${ }^{68}$ Much less is known about the incidence of severe allergic reactions in adults treated with Erwinia after prior severe allergic reactions to the other asparaginases.

\section{Summary}

Asparaginase is a prominent component of therapy in many modern adult ALL treatment protocols, though there are a few exceptions. Recent interest in adapting pediatric regimens for use in young adults has increased asparaginase exposure, especially in this group of patients. Native
L-asparaginase, and now PEG-asparaginase, are regularly employed in frontline treatment of ALL in young adults. As with pediatric patients, severe allergic reactions, thrombosis, pancreatitis, and hepatic injury are prominent with asparaginase use. The incidence of these toxicities increases with age. Erwinia asparaginase is commonly substituted in cases of allergy to L-asparaginase or PEG-asparaginase. In cases of thrombus formation, patients may be successfully retreated with asparaginase once the initial thrombus has been treated. Prophylaxis for thrombus formation may prove to be helpful, but more data are needed. Pancreatitis remains a difficult obstacle to further asparaginase treatment. Given that it precludes most subsequent uses of asparaginase, the diagnosis of pancreatitis must be confirmed by both symptoms and clinical studies. For PEG-asparaginase, an optimal dose and schedule has not been clearly established. An intrapatient dose-escalation trial of PEG-asparaginase might help to guide PEG-asparaginase dosing in adults with ALL.

\section{Disclosure}

The author has served on an advisory board for Sigma Tau Pharmaceuticals.

\section{References}

1. Kidd JG. Regression of transplanted lymphomas induced in vivo by means of normal guinea pig serum. II. Studies on the nature of the active serum constituent: histological mechanism of the regression: tests for effects of guinea pig serum on lymphoma cells in vitro: discussion. J Exp Med. 1953;98:583-606.

2. Broome JD. Evidence that the L-asparaginase of guinea pig serum is responsible for its antilymphoma effects. II. Lymphoma 6C3HED cells cultured in a medium devoid of L-asparagine lose their susceptibility to the effects of guinea pig serum in vivo. J Exp Med. 1963;118:121-148.

3. Hill JM, Roberts J, Loeb E, Khan A, MacLellan A, Hill RW. L-Asparaginase therapy for leukemia and other malignant neoplasms. JAMA. 1967;202:116-122.

4. Beard MEJ, Crowther D, Galton DAG, et al. L-Asparaginase in treatment of acute leukaemia and lymphosarcoma. Br Med J. 1970;1:191-195.

5. Dolowy WC, Henson D, Cornet J, Sellin H. Toxic and antineoplastic effects of L-asparaginase, study of mice with lymphoma and normal monkeys and report on a child with leukemia. Cancer. 1966;19:1813-1819.

6. Oettgen HF, Stephenson PA, Schwartz MK, et al. Toxicity of E. coli L-asparaginase in man. Cancer. 1970;25:253-278.

7. Capizzi RL, Bertino JR, Skeel RT, et al. L-Asparaginase: clinical biochemical, pharmacological and immunological studies. Ann Intern Med. 1971;74:893-901.

8. Haskell CM, Canellos GP, Levental BG, Carbone PP, Serpick AA, Hansen HH. L-asparaginase toxicity. Cancer Res. 1969;29:974-975.

9. Haskell CM, Canellos GP, Levental BG, et al. L-asparaginase: therapeutic and toxic effects in patients with neoplastic disease. $N$ Engl J Med. 1969;281:1028-1034.

10. Asselin BL, Whitin JC, Coppola DJ, Rupp IP, Sallan SE, Cohen HJ. Comparative pharmacokinetic studies of three asparaginase preparations. J Clin Oncol. 1993;11:1780-1786.

11. Asselin BL. The three asparaginases. Comparative pharmacology and optimal use in childhood leukemia. Adv Exp Med Biol. 1999;457: $621-629$. 
12. Avramis VI, Panosyan EH. Pharmacokinetic/pharmacodynamic relationships of asparaginase formulations. The past, the present and recommendations for the future. Clin Pharmacokinet. 2005;44: 367-393.

13. Fu CH, Sakamoto KM. PEG asparaginase. Expert Opin Pharmacother. 2007;8:1977-1984.

14. Kantarjian H, Thomas D, O'Brien S, et al. Long-term follow up results of hyperfractionated cyclophosphamide, vincristine, doxorubicin, and dexamethasone (hyper-CVAD), a dose-intensive regimen, in adult acute lymphocytic leukemia. Cancer. 2004;101:2788-27801.

15. Katarjian HM, Walters RS, Keating MJ, et al. Results of the vincristine, doxorubicin, and dexamethasone regimen in adults with standard- and high-risk acute lymphocytic leukemia. J Clin Oncol. 1990;8: 994-1004.

16. Linker CA, Levitt LJ, O’Donnell M, Forman SF, Ries CA. Treatment of adult acute lymphoblastic leukemia with intensive cyclical chemotherapy: a follow-up report. Blood. 1991;78:2814-2822.

17. Graham ML. Pegaspargase: a review of clinical studies. Adv Drug Deliv Rev. 2003;55:1293-1302.

18. Zeidan A, Wang ES, Wetzler M. Pegasparaginase: where do we stand? Expert Opin Biol Ther. 2009;9:111-119.

19. Tallal L, Tan C, Oettgen H, et al. E. coli L-asparaginase in the treatment of leukemia and solid tumors in 131 children. Cancer. 1970;25: 306-320.

20. Clarkson B, Gaynor J, Franza RB, Furth M. Recent advances in the treatment of acute lymphoblastic leukemia in adults and future prospects. Trans Am Clim Climatol Assoc. 1985;96:41-55.

21. Asselin B. The three asparaginases. comparative pharmacology and optimal use in childhood leukemia. Adv Exp Med Biol. 1999;457: 621-629.

22. Riccardi R, Holcenberg JS, Glaubiger DL, Wood JH, Poplack DG L-Asparaginase pharmacokinetics and asparagine levels in cerebrospinal fluid of rhesus monkeys and humans. Cancer Res. 1981;41(11 Pt 1): 4554-4558.

23. Ahlke E, Nowak-Gottl U, Schulze-Westhoff P. Dose reduction of asparaginase under pharmacokinetic and pharmacodynamic control during induction therapy in children with acute lymphoblastic leukaemia. Br J Haematol. 1997;96:675-681.

24. Pieters R, Hunger SP, Boos J, et al. L-asparaginase treatment in acute lymphoblastic leukemia: a focus on Erwinia asparaginase. Cancer. 2011;117:238-249.

25. Boos J, Werber G, Ahlke E, et al. Monitoring of asparaginase activity and asparagine levels in children on different asparaginase preparations. Eur J Cancer. 1996;32A:1544-1550.

26. Panosyan EH, Seibel NL, Martin-Aragon S, et al. Asparaginase antibody and asparaginase activity in children with higher risk acute lymphoblastic leukemia: Children's Cancer Group Study CCG-1961. J Pediatr Hematol Oncol. 2004;26:333-335.

27. Armstrong JK, Hempel G, Koling S, et al. Antibody against poly(ethylene glycol) adversely affects PEG-asparaginase therapy in acute lymphoblastic leukemia patients. Cancer. 2007;110: 103-111.

28. Schauer P, Arlin ZA, Mertelsmann R, et al. Treatment of acute lymphoblastic leukemia in adults: results of the L-10 and L-10M protocols. $J$ Clin Oncol. 1983;1:462-470.

29. Lister TA, Whitehouse JMA, Beard MEJ, et al. Combination chemotherapy for acute lymphoblastic leukaemia in adults. $\mathrm{Br}$ Med $\mathrm{J}$. 1978;1:199-203.

30. Marcus RE, Catovsky D, Johnson SA, et al. Adult acute lymphoblastic leukaemia: a study of prognostic features and response to treatment over a ten-year period. Br J Cancer. 1986;53:175-180.

31. Hoelzer D, Thiel E, Loffler H, et al. Intensified therapy in acute lymphoblastic and acute undifferentiated leukemia in adults. Blood. 1984;64:38-47.

32. Durrant IJ, Richards SM. Results of Medical Research Council trial UKALL IX in acute lymphoblastic leukaemia in adults: report from the Medical Research Council Working Party on Adult Leukaemia. Br J Haematol. 1993;85:84-92.
33. Radford JE, Jones MP, Gigrich RD, et al. Adult acute lymphoblastic leukemia: results of the Iowa HOP-L protocol. J Clin Oncol. 1989;7: 58-66.

34. Hoelzer D, Thiel H, Loffler H, et al. Prognostic factors in a multicenter study for treatment of acute lymphoblastic leukemia in adults. Blood. 1988;71:123-131.

35. Ellison RR, Mick R, Cuttner J, et al. The effects of postinduction intensification treatment with cytarabine and daunorubicin in adult acute lymphocytic leukemia: a prospective randomized clinical trial by Cancer and Leukemia Group B. J Clin Oncol. 1991;9:2002-2015.

36. Hussein KK, Dahlberg S, Head D, et al. Treatment of acute lymphoblastic leukemia in adults with intensive induction, consolidation, and maintenance chemotherapy. Blood. 1989;73:57-63.

37. Ludwig WD, Rieder H, Bartram CR, et al. Immunopheotypic and genotypic features, clinical characteristics and treatment outcome of adult pro-B acute lymphoblastic leukemia: results of the German multicenter trials GMALL 03/87 and 04/89. Blood. 1998;92:1898-1909.

38. Ribera JM, Ortega JJ, Oriol A, et al. Late intensification chemotherapy has not improved the results of intensive chemotherapy in adult acute lymphoblastic leukemia. Results of a prospective multicenter randomized trial (PETHEMA ALL-89). Haematologica. 1998;83:222-230.

39. Rowe JM, Buck G, Burnett AK, et al. Induction therapy for adults with acute lymphoblastic leukemia: results of more than 1500 patients from the international ALL trial: MRC UKALL XII/ECOG E2993. Blood. 2005;106:3760-3767.

40. Nagura E, Kimura K, Yamada K, et al. Nation-wide randomized comparative study of doxorubicin, vincristine and prednisolone combination therapy with and without L-asparaginase for adult acute lymphoblastic leukemia. Cancer Chemother Pharmacol. 1994;33:359-365.

41. Rijneveld AW, van der Holt B, Daenen SM, et al. Intensified chemotherapy inspired by a pediatric regimen combined with allogeneic transplantation in adult patients with acute lymphoblastic leukemia up to the age of 40. Leukemia. 2011;25:1697-1703.

42. Huguet F, Leguay T, Raffoux E, et al. Pediatric-inspired theraphy in adults with Philadelphia chromosome-negative acute lymphoblastic leukemia: the GRALL-2003 study. J Clin Oncol. 2009;27:911-918.

43. Ribera JM, Oriol A, Sanz MA, et al. Comparison of the results of the treatment of adolescents and young adults with standard-risk acute lymphoblastic leukemia with the Programa Espanol de Tratamiento Hematologia pediatric-based protocol ALL-96. J Clin Oncol. 2008;26: 1843-1849.

44. Labar B, Suciu S, Willemze R, et al. Dexamethasone compared to prednisolone for adults with acute lymphoblastic leukemia or lymphoblastic lymphoma: final results of the ALL-4 randomized, Phase III trial of the EORTC Leukemia Group. Haematologica. 2010;95:1489-1495.

45. Park Young K, Abuchowski A, Davis S, Davis F. Pharmacology of Escherichia coli L-asparaginase polyethylene glycol adduct. Anticancer Res. 1981;1:373-376.

46. Keating MJ, Holmes R, Lerner S, Ho DH. L-Asparaginase and PEG asparaginase-Padt, present, and future. Leuk Lymphoma. 1993;10: $153-157$.

47. Ettinger LJ, Kurtzberg J, Voute PA, Jurgens H, Halpern SL. An open-label, multicenter study of polyethylene glycol-L-asparaginase for the treatment of acute lymphoblastic leukemia. Cancer. 1995;75:1176-1181.

48. Wetzler M, Sandord BL, Kurtzberg J, et al. Effective asparagine depletion with pegylated asparaginase results in improved outcomes in adult acute lymphoblastic leukemia: Cancer and Leukemia Group B study 9511. Blood. 2007;109:4164-4167.

49. Aguayo A, Cortes J, Thomas D, Pierce S, Keating MJ, Kantarjian H. Combination therapy with methotrexate, vincristine, polyethyleneglycol conjugated-asparaginase, and prednisone in the treatment of patients with refractory or recurrent acute lymphoblastic leukemia. Cancer. 1997;86:1203-1209.

50. Rosen O, Muller HJ, Gokbuget N, et al. Pegylated asparaginase in combination with high-dose methotrexate for consolidation in adult acute lymphoblastic leukemia in first remission: a pilot study. Br J Hematol. 2003;123:836-841. 
51. Douer D, Yampolsky H, Cohen LJ, Watkins K, Periclou AP, Avramis VI. Pharmacodynamics and safety of intravenous pegaspargase during remission induction in adults aged 55 years or younger with newly diagnosed acute lymphoblastic leukemia. Blood. 2007;109:2744-2750.

52. Rytting M, Earl M, Douer D, Muriera B, Advani A, Bleyer A. Toxicities in adults with acute lymphoblastic leukemia (ALL) treated with regimens using pegasparaginase. Blood. 2008;112:Abstract 1924.

53. Avramis VI, Sencer S, Periclou AP, et al. A Randomized comparison of native Escherichia coli asparaginase and polyethylene glycol conjugated asparaginase for treatment of children with newly diagnosed standard-risk acute lymphoblastic leukemia: a children's cancer group study. Blood. 2002;99:1986-1994.

54. Kearney SL, Dahlberg SE, Levy DE, Voss SD, Sallan SE, Silverman LB. Clinical course and outcome in children with acute lymphoblastic leukemia and asparaginase-associated pancreatitis. Pediatr Blood Cancer. 2009;53:162-167.

55. Freund M, Diedrich H, Ganser A, et al. Treatment of relapsed or refractory adult acute lymphocytic leukemia. Cancer. 1992;69:709-716.

56. Grace RF, Dahlberg SE, Neuberg D, et al. The frequency and management of asparaginase-related thrombosis in pediatric and adult patients with acute lymphoblastic leukemia treated on Dana-Farber Cancer Institute consortium protocols. Br J Haematol. 2011;152:452-459.

57. Appel IM, Kazemier KM, Boos J, et al. Pharmacokinetic, pharmacodynamic and intracellular effects of PEG-asparagines in newly diagnosed childhood acute lymphoblastic leukemia: results from a single agent window study. Leukemia. 2008;22:1665-1679.

58. Wenner KA, Pinheiro JP, Escherich G, et al. Asparagine concentration in plasma after 2,500 IU/m(2) PEG-asparaginase i.v. in children with acute lymphoblastic leukemia. Klin Padiatr. 2005;217:321-326. German.

59. Dinnorf PA, Gootenbert J, Cohen M, Keegan P, Pazdur R. FDA drug approval summary: Pegaspargase (Oncaspar) for the first-line treatment of children with acute lymphoblastic leukemia. Oncologist. 2007;12:991-994.
60. Nowak-Gotti U, Kenet G, Mitchell LG. Thrombosis in childhood acute lymphoblastic leukemia: epidemiology, aetiology, diagnosis, prevention and treatment. Best Pract Res Clin Haematol. 2009;22:103-114.

61. Hunault-Berger M, Chevallier P, Delain M, et al. Changes in antithrombin and fibrinogen levels during induction chemotherapy with L-asparaginase in adult patients with acute lymphoblastic leukemia or lymphoblastic lymphoma. Use of supportive coagulation therapy and clinical outcome: the CAPELAL study. Haematologica. 2008;93: 1488-1494.

62. Hernández-Espinosa D, Minano A, Ordonez A, et al. Dexamethasone induces a heat-stress response that ameliorated the conformational consequences on antithrombin of L-asparagines treatment. J Thromb Haemost. 2009;7:1128-1133.

63. Durden DL, Salazar AM, Distasio JA. Kinetic analysis of hepatotoxicity associated with antieoplastic asparaginases. Cancer Res. 1983;43: 1602-1605.

64. Linker C, Damon L, Ries C, Navarro W. Intensified and shortened cyclical chemotherapy for adult acute lymphoblastic leukemia. J Clin Oncol. 2002;20:2464-2471.

65. Chang J, Medlin S, Kahl B, et al. Augmented and standard BerlinFrankfurt-Munster chemotherapy for treatment of adult acute lymphoblastic leukemia. Leuk Lymphoma. 2008;49:2298-2307.

66. Larson RA, Fretzin MH, Dodge RK, Schiffer CA. Hypersensitivity reactions to L-asparaginase do not impact on the remission duration of adults with acute lymphoblastic leukemia. Leukemia. 1998;12:660-665.

67. Storring JM, Minden MD, Kao S, et al. Treatment of adults with BCRABL negative acute lymphoblastic leukemia with a modified pediatric regimen. Br J Hematol. 2009;146:76-85.

68. Vrooman LM, Supko JG, Neuberg DS, et al. Erwinia asparaginase after allergy to E. coli asparaginase in children with acute lymphoblastic leukemia. Pediatr Blood Cancer. 2010;54:199-205.
Blood and Lymphatic Cancer: Targets and Therapy

\section{Publish your work in this journal}

Blood and Lymphatic Cancer: Targets and Therapy is an international, peer-reviewed, open access journal focusing on blood and lymphatic cancer research, identification of therapeutic targets and the optimal use of preventative and integrated treatment interventions to achieve improved outcomes, enhanced survival and quality of life for the cancer

\section{Dovepress}

patient. The manuscript management system is completely online and includes a very quick and fair peer-review system. Visit http://www. dovepress.com/testimonials.php to read real quotes from published authors. 